\title{
Effect of Quercetin in the UV-Irradiated Human Keratinocyte HaCaT Cells and A Model of Its Binding To p38 MAPK
}

\author{
Hum Nath Jnawali, Eunjung Lee, Areum Shin, Young Guen Park, and Yangmee Kim* \\ Department of Bioscience and Biotechnology, Bio-Molecular Informatics Center, Institute of KU Biotechnology, \\ Konkuk University, Seoul 143-701, Korea. ${ }^{*}$ E-mail: ymkim@konkuk.ac.kr \\ Received May 20, 2014, Accepted May 29, 2014
}

\begin{abstract}
Quercetin is a major dietary flavonoid found in onions, apples, tea, and red wine, and potentially has beneficial effects on disease prevention. We carried out this study to investigate the effect of quercetin on UVB-induced matrix metalloproteinase-1 (MMP-1) expression in human keratinocyte HaCaT cells and to further understand the mechanisms of its action. The anti-inflammatory activity of quercetin was investigated and quercetin significantly suppressed the NO production in LPS-stimulated RAW264.7 mouse macrophages. Post treatment of quercetin decreased UV irradiation-induced phosphorylation of JNK, p38 MAPK, and ERK by $91 \%, 21 \%$, and $17 \%$, respectively. MMP-1 is mainly responsible for the degradation of dermal collagen during the aging process of human skin and quercetin suppressed the UVB-induced MMP-1 by $94 \%$. Binding studies revealed that quercetin binds to $\mathrm{p} 38$ with high binding affinity $\left(1.85 \times 10^{6} \mathrm{M}^{-1}\right)$. The binding model showed that the $4^{\prime}-$ hydroxy groups of the B-ring of quercetin participated in hydrogen bonding interactions with the side chains of Lys53, Glu71, and Asp168 and the 5-hydroxy group of the A-ring formed a hydrogen bond with the backbone amide of Met109. The major finding of this study shows that quercetin inhibits phosphorylation of JNK, p38 MAPK, and ERK pathway leading to the prevention of MMP-1 expression in human keratinocyte $\mathrm{HaCaT}$ cells. Therefore, our findings suggested the potentials of quercetin as a skin anti-photoaging agent.
\end{abstract}

Key Words : Flavonoid, Quercetin, Anti-inflammatory activity, Binding model, UV irradiation

\section{Introduction}

Ultraviolet (UV) irradiation is one of the most ubiquitous environmental hazards that impact every organism under the sun. Chronic exposure to UV irradiation causes sunburn, DNA damage, immune suppression, and connective tissue degradation. ${ }^{1}$ Exposure of mammalian cells to UV irradiation gives rise to two main alterations that lead to harmful outcomes: chemical modifications to DNA, and generation of reactive oxygen species. Generation of reactive oxygen is the cause of UV irradiation-induced oxidative stress. Severe oxidative stress results in apoptotic or necrotic cell death, ${ }^{2}$ while sub-lethal oxidative stress activates multiple intracellular signal transduction pathways. ${ }^{3}$ Activation of cell surface receptors by UV irradiation stimulates signal transduction pathways involving MAP kinase family members, including ERK, JNK, and $\mathrm{p} 38$, which lead to activation of the activator protein-1 (AP-1) transcription factor family members. ${ }^{4}$ Another important transcription factor family activated in response to UV irradiation is nuclear factor kappa $\mathrm{B}(\mathrm{NF}-\mathrm{\kappa B}) .^{5}$ It has been reported that antioxidant genistein protect against the UV radiation-induced inflammation. ${ }^{6}$

Exogenous supplementation of antioxidants has been

Abbreviations: AP-1, activator protein-1; ERK, extracellular signalregulated kinase; JNK, Jun-N terminal kinase; LPS, lipopolysaccharide; GAPDH, glyceraldehyde 3-phosphate; MAPK, mitogen-activated protein kinase; MMP-1, matrix metalloproteinases-1; NO, nitric oxide; NF-kB, nuclear factor kappa B; UV, ultraviolet shown to prevent UV irradiation-induced oxidative damage, ${ }^{7}$ yet their effects on signaling pathways leading to gene expression are not well understood. Recent investigations suggest that tyrosine kinase and MAP kinase pathway might be potential pharmacological targets. ${ }^{8}$ Flavonoids are low molecular weight compounds rich in seeds, citrus fruits, red wine, tea, and olive oil. Flavonoids exert several biological activities, which are mainly related to their ability to inhibit enzymes and/or to their antioxidant properties, and are able to regulate the immune response. ${ }^{9}$ Quercetin $(3,3 ', 4,5,7-$ pentahydroxyflavone) (Figure 1) one of the abundant flavonoltype flavonoids found in onions and other vegetables, has been reported to possess various pharmacological properties, including acting as an antioxidant and inhibiting carcinogenesis and allergies. ${ }^{10-13}$ Molecular mechanisms involved in the anti-inflammatory effects of quercetin are widely studied. However, the effects of quercetin in the UV irradiated human keratinocyte $\mathrm{HaCaT}$ cells has not been studied, yet.

In this study, we examined the effects and mode of action of quercetin in the UV irradiated human keratinocyte $\mathrm{HaCaT}$ cells. For the first time, we investigated the interactions between quercetin with $\mathrm{p} 38$ MAPK using molecular-docking and fluorescence-quenching studies. These results suggested that quercetin can be a potent agent with anti-inflammatory agents as well as a skin photoaging agent.

\section{Materials and Methods}

Reagents. Quercetin (Figure 1) was purchased from Sigma- 


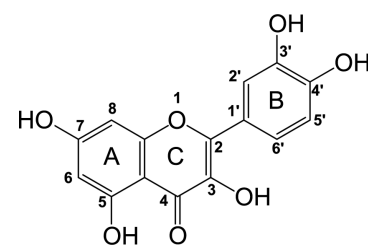

Figure 1. Structure of quercetin.

Aldrich (St. Louis, MO, USA) and dissolved in DMSO at 10 $\mathrm{mg} / \mathrm{mL}$ for the stock solution.

Docking Study. Using the X-ray crystallography structure of p38 MAPK (3S3I.pdb), we defined the ATP-binding site of p38. Quercetin was docked to p38 using CDOCKER, a CHARMm-based molecular dynamics (MD) method for ligand-docking, in Discovery Studio modeling (Accelrys Inc., San Diego, USA). This algorithm assumes a rigid protein and permits only the ligand to be flexible. The Input Site Sphere parameter specifies a sphere around the center of the binding site, where the CDOCKER experiment is to be performed. The center of the sphere is used in the CDOCKER algorithm for initial ligand placement. The MD simulated annealing process is performed using a rigid protein and flexible ligand. The final minimization step is applied to the ligand's docking pose. The minimization consists of 50 steps of steepest descent followed by up to 200 steps of conjugated-gradient using an energy tolerance of $0.001 \mathrm{kcal} / \mathrm{mol}^{15}$

Fluorescence Quenching Analysis. Experiments were performed at $25{ }^{\circ} \mathrm{C}$ on an RF-5301PC spectrofluorophotometer (Shimadzu, Kyoto, Japan). We titrated quercetin to 10 $\mu \mathrm{M}$ p38 protein solution in $50 \mathrm{mM}$ sodium phosphate buffer containing $100 \mathrm{mM} \mathrm{NaCl}$ at $\mathrm{pH} 8.0$, with a final protein: inhibitor ratio of 1:10. The sample was placed in a $2 \mathrm{~mL}$ cuvette, with excitation and emission path lengths of $10 \mathrm{~nm}$. Using tryptophan emission, we determined the fluorescence quantum yields of $\mathrm{p} 38$ and the ligand. The detailed methods are provided in a previous article. ${ }^{16}$

Quantification of Nitrite Production in LPS-stimulated RAW264.7 Cells. The mouse macrophage-derived RAW264.7 cell line was purchased from the American Type Culture Collection (Manassas, VA, USA). Nitrite accumulation in culture media was used as an indicator of NO production. ${ }^{17}$ Raw264.7 cells were plated at a density of $1 \times 10^{5}$ cells $/ \mathrm{mL}$ and stimulated with LPS $(20 \mathrm{ng} / \mathrm{mL})$ from Escherichia coli O111:B4 (Sigma-Aldrich) in the presence or absence of quercetin for $24 \mathrm{~h}$. Isolated supernatant fractions were mixed with an equal volume of Griess reagent $(1 \%$ sulfanilamide, $0.1 \%$ naphthylethylenediamine dihydrochloride, and $2 \%$ phosphoric acid) and incubated at room temperature for 10 min. Nitrite production was determined by measuring absorbance at $540 \mathrm{~nm}$ and was converted to nitrite concentration by reference to a standard curve generated with $\mathrm{NaNO}_{2} .^{17}$

UVB Irradiation. The human keratinocyte $\mathrm{HaCaT}$ cells were purchased from the American Type Culture Collection (Manassas, VA, USA). HaCaT cells were cultured in Dulbecco's modified Eagle's medium (DMEM; GibcoBRL,
Grand Island, NY, USA) supplemented with 5\% heat inactivated fetal bovine sereum (FBS; Hyclone, Logan, UT, USA) and were grown to near confluency. Before UVB irradiation, the medium was removed from the culture plates and the cells were washed twice with phosphate-buffered saline (PBS) and then covered with a thin layer of PBS. This was followed by $40 \mathrm{~mJ} / \mathrm{cm}^{2}$ total energy dose of UVB irradiation. PBS-washed cells not exposed to UV irradiation served as controls. The UVB light source was a bank of four FS24T12-UVB-HO sunlamps equipped with a UVB Spectra 305 Dosimeter (Daavlin Co., Bryan, OH), which emitted $\sim 80 \%$ radiation in the range $280-340 \mathrm{~nm}$ with a peak emission at $314 \mathrm{~nm}$, as monitored with a SEL 240 photodetector, 103 filter and 1008 diffuser attached to an IL1400A Research Radiometer (International Light, Newburyport, MA). The UVB irradiation doses were also calibrated using an IL1400A radiometer. After irradiation, the cells were further incubated in culture medium for various times and harvested with a cell scraper.

Western Blotting. Protein was isolated from $\mathrm{HaCaT}$ cells in the presence or absence of UV at $20 \mu \mathrm{M}$ quercetin. Proteins were then detected as described previously ${ }^{18}$ using the following antibodies: phospho-ERK (1:2000; Cell Signaling Technology, Beverly, MA, USA), phospho-p38 (1:2000, Cell Signaling Technology), phospho-JNK (1:1000, Cell Signaling Technology), and $\beta$ actin (1:5000, Sigma-Aldrich, St. Louis, MO, USA). The relative amount of protein associated with each antibody was quantified using ImageJ (NIH, Bethesda, USA).

\section{Results and Discussion}

Docking Study. To know the binding model of quercetin and $\mathrm{p} 38$, we carried out docking study with quercetin and p38. Quercetin was docked into the ATP-binding active site of $\mathrm{p} 38$. We investigated the importance of the hydrogen bonds that participate in the interaction between quercetin and p38 (Figure 2). The side chains of Lys53, Glu71, and Asp168 formed a network of hydrogen bonds with the 4'hydroxy groups of the B-ring of quercetin, while the 5hydroxy group of the A-ring formed a hydrogen bond with the backbone amide of Met109 (Figure 2). Quercetin formed additional hydrophobic interactions with Val38 and Ala51 in the B-ring and Ala157 and Leu167 in the A-ring of p38 MAKP, as shown in Figure 2. The results of this docking study should provide helpful insights in understanding the mechanism by which quercetin inhibits p38 MAPKs.

Fluorescence Quenching. The p38 protein contains five tryptophan residues at positions 18, 187, 197, 207, and 337. Among these, Trp18 as shown in Figure 2(b) is in closest proximity to the substrate binding site of $\mathrm{p} 38$ and protein fluorescence should be decreased upon ligand binding to p38. Fluorescence quenching was used to estimate the binding constant, $K$. The binding (or dissociation) constant, $K$, is defined as [complex]/[free protein][free inhibitor]. ${ }^{19}$ We successfully analyzed the binding affinities between $\mathrm{p} 38$ and quercetin using this method. Fluorescence titration curves 
(a)

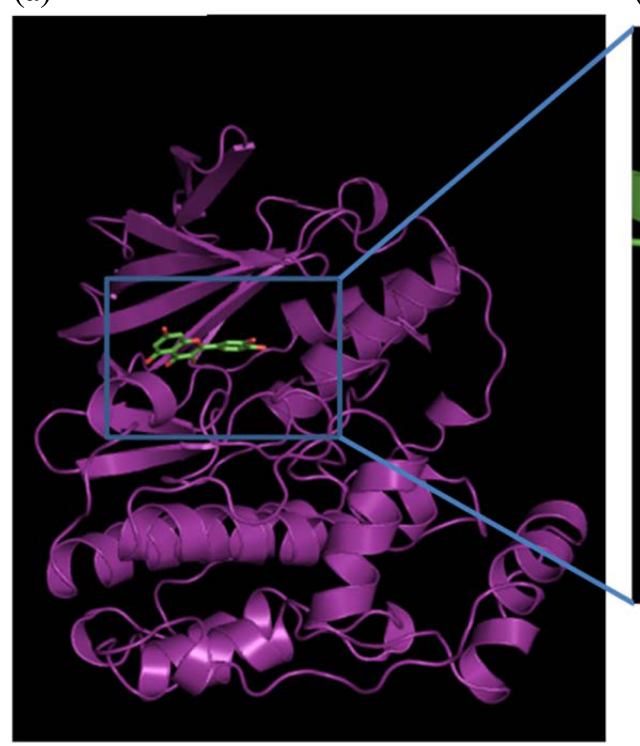

(b)

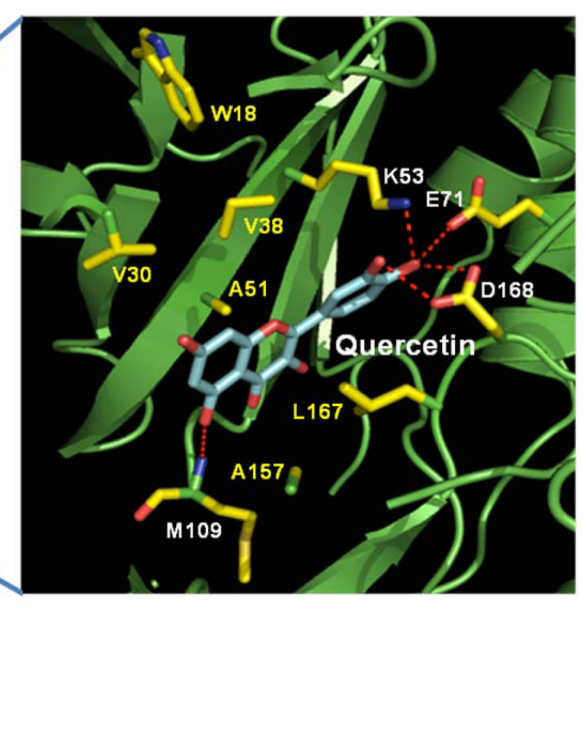

Figure 2. (a) Overall structure of human p38 in complex with quercetin and ATP-binding site was marked with blue box. (b) In the closeup view, binding model of quercetin and p38 determined by docking study is shown. Red dotted line presented hydrogen bonds between p38 and quercetin.

for p38 with quercetin are depicted in Figure 3. The fluorescence intensity changed with increasing concentrations of quercetin. This spectral change was attributed to the formation of protein- quercetin complexes. As shown in Figure 3, a significant decrease was evident in the quenching of p38 tryptophan fluorescence in the presence of quercetin. The binding affinity of the quercetin was estimated to be $1.85 \times$ $10^{6} \mathrm{M}^{-1}$.

Effects of Quercetin on Nitrite Production in LPS-stimulated RAW264.7 Cells. NO, a free radical that plays a pivotal role in cell survival and death, exerts various proinflammatory effects on many cell types. ${ }^{20}$ Studies of diverse inflammatory diseases, including cardiovascular conditions, have shown that high levels of NO in inflamed tissue are

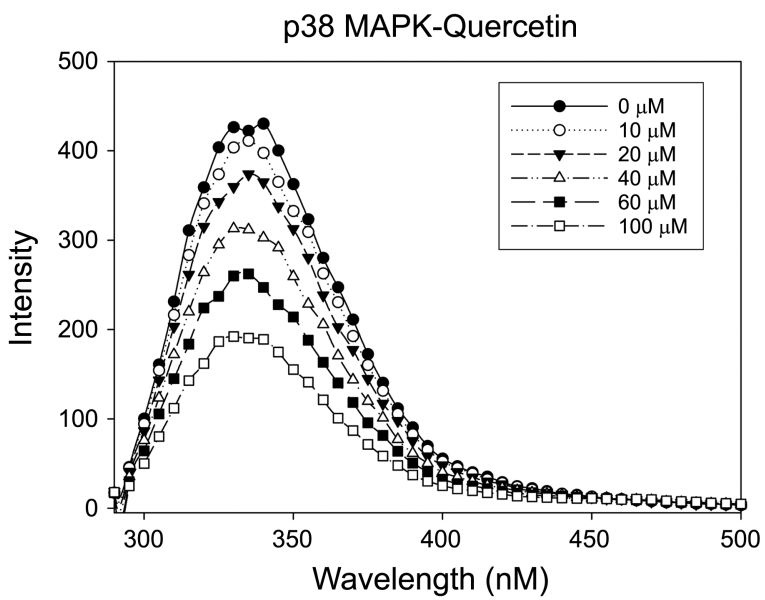

Figure 3. Fluorescence spectra of an aqueous solution of $10 \mu \mathrm{M}$ p38 in the presence of different concentrations of quercetin $(0,10$, $20,40,60$, and $100 \mu \mathrm{M})$ at $\mathrm{pH} 7.0$. Samples were excited at 290 $\mathrm{nm}$, and emission spectra recorded for light scattering effects from 290 to $500 \mathrm{~nm}$. cytotoxic $^{21}$ and lead to cell death. ${ }^{22}$ To know the effects of quercetin that inhibits with LPS signaling and inflammatory cascade, we investigated nitrite production in LPS-stimulated RAW264.7. Here, the effects of different concentrations of quercetin $(1,2.5,5,10$, and $20 \mu \mathrm{M})$ were investigated on LPS-induced NO production in RAW264.7 cells by measuring the accumulation of nitrite in the culture medium. As expected, LPS stimulation increased the concentration of NO significantly. Notably, treatment with 10 and $20 \mu \mathrm{M}$ quercetin decreased LPS-induced NO production completely (Figure 4).

Effects of Quercetin on the UVB-Induced Phosphorylation of MAPKs. To evaluate the inhibitory effect of quer-

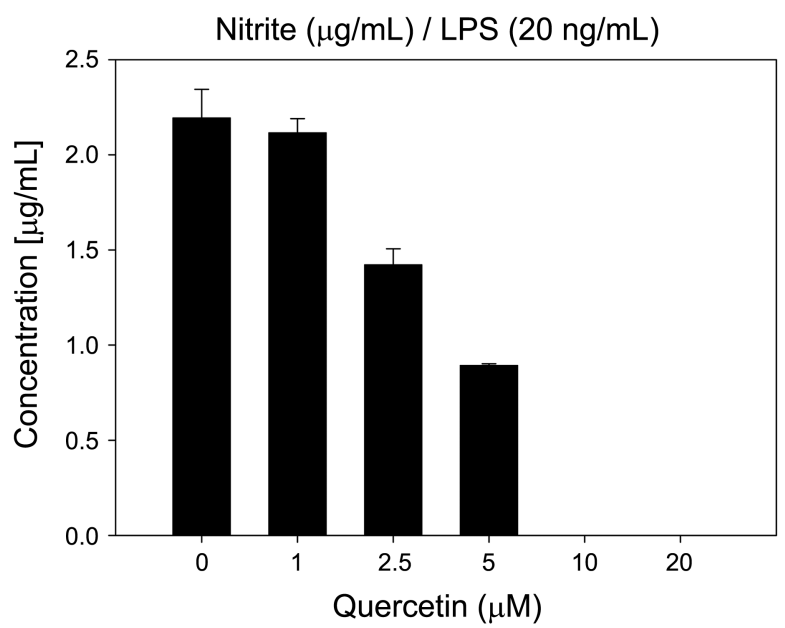

Figure 4. Inhibition of NO production by quercetin in LPSstimulated RAW264.7 cells. RAW264.7 cells were treated with quercetin $(1,2.5,5,10$ and $20 \mu \mathrm{M})$ in the presence of LPS $(20$ $\left.\mathrm{ng} \cdot \mathrm{mL}^{-1}\right)$ for $24 \mathrm{~h}$. The error bars represent standard deviation values of mean determined from three independent experiments. 


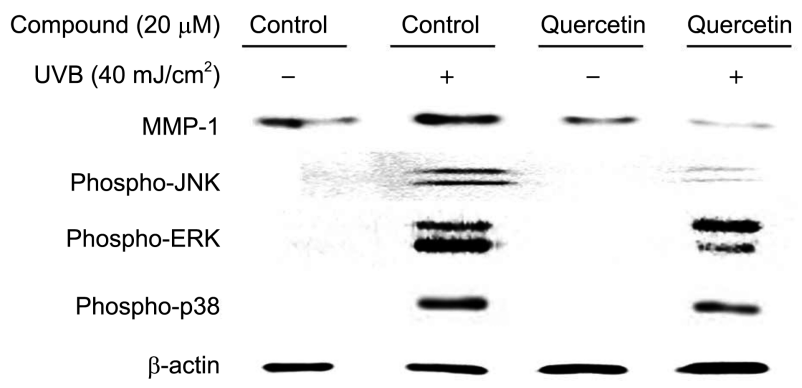

Figure 5. Effects of quercetin on the UVB-induced MMP-1, phospho-JNK, phospho-ERK, phospho-p38, and $\beta$-actin in human keratinocyte HaCaT cells. MMP-1, phospho-JNK, phospho-ERK, phospho-p38, and $\beta$-actin protein levels were determined by western blot analysis using specific antibodies. The relative protein expression was quantified using ImageJ (NIH, Bethesda, MD, USA).

cetin on UVB-induced phosphorylation of members of the MAPK family such as ERK, p38, and JNK, the expression of their phosphorylated forms was examined by using western blotting. It has been reported that UV-irradiated fibroblasts elevate the expression of phosphorylated MAPKs family. ${ }^{4}$ As shown in Figure 5, quercetin suppressed the UVBinduced phosphorylation of JNK, p38 MAPK, and ERK by $91 \%, 21 \%$, and $17 \%$, respectively (Figure 5). Activation of cell surface receptors by UV irradiation stimulates signal transduction pathways involving these MAP kinase family members. We observed that quercetin markedly inhibited UVB-induced activation of JNK and weakly by p38 MAPK, and ERK.

Effects of Quercetin on the UVB-Induced MMP-1. Matrix metalloproteinases (MMPs) are AP-1 target genes that play important roles in skin cancer and photo-aging. ${ }^{23}$ MMPs are a family of zinc-dependent endopeptidases that include matrix metalloproteinases-1 (MMP-1; interstitial collagenase), MMP-3 (stromelysin-1), and MMP-9 (92-kDa type IV gelatinase). In particular, MMP-1 is mainly responsible for the degradation of dermal collagen during the aging process of human skin. ${ }^{24-26}$ As shown in Figure 5, quercetin suppressed the UVB-induced MMP-1 by $94 \%$.

\section{Conclusion}

In our study, post treatment of quercetin exhibits inhibitory effect on MMP-1 and ERK, p38, and JNK phosphorylation in UV-induced human keratinocyte HaCaT cells. We observed quercetin markedly inhibited JNK's phosphorylation and MMP-1 by $91 \%$ and $94 \%$ respectively, while the phosphorylation of p38 and ERK by $21 \%$ and $17 \%$, respectively (Figure 5). Here, we demonstrated the inhibitory effect of quercetin on the production of the pro-inflammatory mediators NO in the LPS-activated macrophage culture system and found that quercetin thoroughly inhibits NO production in LPS-stimulated macrophages at $10 \mu \mathrm{M}$ concentrations. Quercetin, with good anti-inflammatory activities, showed high binding affinity to $\mathrm{p} 38$ at $1.85 \times 10^{6} \mathrm{M}^{-1}$. From the docking study, a binding model of quercetin and p38 was proposed.
The side chains of Lys53, Glu71, and Asp168 formed hydrogen bonds with the 4'-hydroxy groups of the B-ring of quercetin, while the 5-hydroxy group of the A-ring formed a hydrogen bond with the backbone amide of Met109 (Figure 2). Therefore, our results strongly suggested that quercetin can be a potential therapeutic agent for the prevention and the treatment of skin photoaging.

Acknowledgments. This work was supported by a grant from the Priority Research Centers Program (2009-0093824) and Basic Science Research Program (2013R1A1A2058021), through the National Research Foundation of Korea, funded by the Ministry of Education, Science and Technology.

\section{References}

1. Ichihashi, M.; Ueda, M; Budiyanto, A; Bito, T.; Oka, M.; Fukunaga, M.; Tsuru, K.; Horikawa, T. Toxicology 2003, 189, 21.

2. Bertling, C.; Lin, F.; Girotti, A. Photochem. Photobiol. 1996, 64, 137.

3. Rosette, C.; Karin, M. Science 1996, 274, 1194.

4. Xu, Y. R.; Fisher, G. J. J. Dermatol. Sci. 2005, 1, S1.

5. Cooper, S. J.; Bowden, G. T. Curr. Cancer Drug Targets 2007, 7, 325.

6. Widyarini, S.; Spinks, N.; Husband, A. J.; Reeve, V. E. Photochem. Photobiol. 2001, 74, 465.

7. Kang, S.; Chung, J.; Lee, J.; Fisher, G.; Wan, Y.; Duell, E.; Voorhees, J. J. J. Invest. Dermatol. 2003, 120, 835.

8. Cho, S.; Park, S.; Kwon, M.; Jeong, T.; Bok, S.; Choi, W.; Jeong, W. I.; Ryu, S. Y.; Do, S. H.; Lee, C. S.; Song, J. C.; Jeong, K. S. Mol. Cell. Biochem. 2003, 243, 153.

9. Hollman, P. C.; de Vries, J. H.; Van Leeuwen, S. D.; Mengelers, M. J.; Katan, M. B. Am. J. Clin. Nutr. 1995, 62, 1276.

10. Paganga, G.; Miller, N.; Rice-Evans, C. A. Free Radic. Res. 1999, $30,153$.

11. Murota, K.; Mitsukuni, Y.; Ichikawa, M.; Tsushida, T.; Miyamoto, S.; Terao, J. J. Agric. Food Chem. 2004, 52, 1907.

12. Kuo, P. C.; Liu, H. F.; Chao, J. I. J. Biol. Chem. 2004, 279, 55875.

13. Knekt, P.; Kumpulainen, J.; Jarvinen, R.; Rissanen, H.; Heliovaara, M.; Reunanen, A.; Hakulinen, T.; Aromaa, A. Am. J. Clin. Nutr. 2002, 76, 560

14. Janssen-Heininger, Y.; Poynter, M.; Baeuerle, P. A. Free Radic. Biol. Med. 2000, 28, 1317.

15. Vieth, M.; Hirst, J. D.; Dominy, B. N.; Daigler, H.; Brooks, C. L. J. Comput. Chem.1998, 19, 1623.

16. Lee, J. Y.; Jeong, K. W.; Lee, J. U.; Kang, D. I.; Kim, Y. Bioorg. Med. Chem. 2009, 17, 1506.

17. Green, L. C.; Wagner, D. A.; Glogowski, J.; Skipper, P. L.; Wishnok, J. S.; Tannenbaum, S. R. Anal. Biochem. 1982, 126, 131.

18. Jung, H. W.; Mahesh, R.; Park, J. H.; Boo, Y. C.; Park, K. M.; Park, Y.-K. Int. Immunopharmacol. 2010, 10, 155.

19. Möller, M.; Denicola, A. Biochem. Mol. Biol. Educ. 2002, 30, 309.

20. Albina, J. E.; Reichner, J. S. New Horiz. 1995, 3, 46.

21. Llorens, S.; Nava, E. Curr. Vasc. Pharmacol. 2003, 1, 335.

22. Tripathi, P.; Tripathi, P.; Kashyap, L.; Singh, V. FEMS Immunol. Med. Microbiol. 2007, 51, 443.

23. Fisher, G.; Datta, S.; Talwar, H.; Wang, Z.; Varani, J.; Kang, S.; Voorhees, J. J. Nature 1996, 379, 335.

24. Visse, R.; Nagase, H. Circ. Res. 2003, 92, 827.

25. McCawley, L. J.; Matrisian, L. M. Curr. Opin. Cell Biol. 2001, 13, 534.

26. Brennan, M.; Bhatti, H.; Nerusu, K. C.; Bhagavathula, N.; Kang, S.; Fisher, G. J.; Varani, J.; Voorhees, J. J. Photochem. Photobiol. 2003, 78, 43 . 